\title{
Evaluation and planning of Chagas control activities using geospatial tools
}

\author{
Diego Weinberg, ${ }^{1}$ Mario Lanfri, ${ }^{2}$ Carlos M. Scavuzzo, ${ }^{2}$ Marcelo Abril, ${ }^{1}$ Sofía Lanfri ${ }^{1}$ \\ ${ }^{1}$ Mundo Sano Foundation, Buenos Aires; ${ }^{2}$ National Commission for Space Activities, Córdoba, Argentina
}

\begin{abstract}
Chagas continues to be a relevant public health problem in Latin America. In this work, we present a spatiotemporal analysis applied for the evaluation and planning of Chagas vector control strategies. We analysed the spatial distribution of the vector Triatoma infestans infestation related to ongoing control interventions cycles in rural communities near Añatuya, Santiago del Estero, Argentina. A geographical information system was developed for the spatial analysis obtaining, for each house, variables that describe the history of spraying and infestation at each time of interventions. Bi-dimensional histograms were used to describe the spatiotemporal pattern of these activities and peri-domestic infestation at the last intervention was modelled by a neural network model. We qualitatively evaluate control programmes considering the history of infestation and spraying from a spatiotemporal point of view, incorporating new ways of visualising this information. Predictions are based on novel, non-linear models and spatiotemporal indices, which should be useful for strategical-
\end{abstract}

Correspondence: Diego Weinberg, Mundo Sano Foundation, Paraguay 1535, C1061 ABC, Buenos Aires, Argentina.

Tel. +54.11.4872.1333 - Fax: +54.11.4872.1334.

E-mail: dweinberg@mundosano.org

Key words: Chagas vector control; Spatiotemporal analysis; Geographical information systems; Neural network model; Argentina.

Acknowledgements: we would like to acknowledge the National Ministry of Health (Argentina), the Vector-Borne Transmission Direction from Santiago del Estero, and the Municipality of Añatuya (Santiago del Estero). We would also like to thank Dr. Oscar Ledesma Patiño.

Conflict of interest: the authors declare no potential conflict of interest.

Funding: resources from the Mundo Sano Foundation.

Received for publication: 15 May 2019.

Revision received: 2 September 2019.

Accepted for publication: 5 October 2019.

${ }^{\circ}$ Copyright: the Author(s), 2019

Licensee PAGEPress, Italy

Geospatial Health 2019; 14:786

doi:10.4081/gh.2019.786

This article is distributed under the terms of the Creative Commons Attribution Noncommercial License (CC BY-NC 4.0) which permits any noncommercial use, distribution, and reproduction in any medium, provided the original author(s) and source are credited. ly allocating Chagas control resources in the future and thus help to better plan spraying strategies.

\section{Introduction}

American trypanosomiasis or Chagas is one of the most serious, endemic parasitic diseases of Latin America (Moreno et al., 2012) with social and economic impacts exceeding the combined effects of other parasitic diseases such as malaria, leishmaniasis and schistosomiasis (Dias and Schofield, 1999). It is caused by the protozoan parasite Trypanosoma cruzi with Triatoma infestans (Hemiptera, Reduviidae, Triatominae) as its main vector in the southern cone of South America. The insect, known by different local names as kissing bug, vinchuca or chinche, has spread Chagas across 21 Latin American countries causing more deaths than any other parasitic disease (WHO, 2003). Moreover, the World Health Organization (WHO) estimates that more than six million people are infected by T. cruzi (WHO, 2016).

Chagas is one of a group of neglected and tropical diseases or diseases of poverty (WHO, 2019) with multiple socioeconomic and environmental determinants. Coexistence of triatomine vectors, mammal reservoirs and exposed people is thus found in housings of precarious quality (mainly in rural and peri-urban zones), located in areas characterized by poverty with social instability and/or high rates of migration and common in groups linked with the seasonal work, e.g. harvesting - all circumstances outside the jurisdiction of the health sector. Moreover, at the community level Chagas often coincides with the transmission of other infectious diseases like tuberculosis, malaria and other neglected diseases together with chronic malnutrition, thereby contributing significantly to the deterioration of the general health of that community.

In the last decades, Chagas vector control programmes, mainly based on intra- and peri-domestic spraying with pyrethroid insecticides (Dias and Schofield, 1999; Silveira and Vinhaes, 1999), in the Southern Cone Initiative countries (Argentina, Bolivia, Brazil, Chile, Paraguay, and Uruguay) (Dias, 2002) reduced the impact in about $80 \%$ of the original distribution area of the T. infestans vector (Moreno et al., 2012). The affected population in Latin America decreased from around 20 millions in the 1980 s to less than 10 million during the first years of 2000 (Remme et al., 2006). Wide areas of South and Central America are now certified by the Pan American Health Organization as having interrupted the transmission of $T$. cruzi by all ochthonous, triatomine vectors (Schofield et al., 2006; Abad-Franch et al., 2013; Gorla et al., 2015).

Although surveillance and control $(\mathrm{S} \& \mathrm{C})$ remains the main preventive intervention against Chagas in endemic countries (Waleckx et al., 2015) such programmes have not been successful in the Gran Chaco region (that covers western Paraguay, northwestern Argentina and eastern Bolivia) (Moreno et al., 2012), particularly not in in the province of Santiago del Estero in Argentina, 
where Chagas is highly endemic and the T. infestans populations seem to be the most resilient to vector chemical control interventions (Gürtler et al., 2004). The unsustainable systematic vector control interventions derived from instabilities of health policies, the very low efficacy of suspension concentrate pyrethroid formulation used to eliminate $T$. infestans populations infesting peridomestic structures and the occurrence of pyrethroid-resistant insect populations can be mentioned as responsible factors (Gorla et al., 2015). More than 50 years of vector control in this region demonstrates that vertically coordinated spraying campaigns aiming at full coverage are unsustainable in practice. Understaffed programmes are challenged by the number of households and the vast extension of the endemic area make insecticide coverage limited and results short-lived. Our rationale was to recognize this inherent limitation and provide a scientific base for the improvement of current interventions aimed at an analysis of the spatial distribution of $T$. infestans infestation in rural communities and its relation to the control intervention cycle.

\section{Materials and Methods}

\section{Background}

Santiago del Estero is the Argentinean province with the highest Chagas prevalence, i.e. at least 200.000 infected people (Abril et al., 2009). This province presents the conjunction of three relevant factors: the parasite, the vector and humans living under cultural, social and environmental conditions favourable for this infection, such as houses (ranchos) built of adobe, mud, wooden sticks and straw with limited sanitary possibilities.

In February 2005, Mundo Sano Foundation (Fundacion Mundo Sano, FMS), a nongovernmental foundation in Argentina working for the prevention and control of communicable diseases, introduced the Integral Chagas Program (IChP), which consists of complementary approaches supporting the ongoing traditional practices of vector control in Añatuya, Santiago del Estero. IChP encourages community participation in activities aimed at interrupting vector domicile transmission by i) surveillance and control (S\&C) of T. infestans based on spraying with pyrethroid insecticides; and ii) improving the quality of dwellings and the peridomestic situation (Abril et al., 2009; Weinberg et al., 2018). Verbal consent is obtained from the responsible adult dwellers in order to join the programme.

The house improvement subprogramme, described by Weinberg et al. (2018), includes actions meant to reduce triatomine infestation by: i) waterproofing of roofs, walls and ceilings includ- ing washing of walls externally and internally; and ii) peri-domestic organization including enhancing structures, such as goat pens and chicken coops. Improvements in each household were always carried out by the community in a solidarity manner, where, those unable to improve their own house were helped by other community members (Abril et al., 2009). After this, all houses had similar conditions with respect to vulnerability to triatomines.

Geographical information systems (GIS), a set of methods and tools for collecting, storing, retrieving, transforming and displaying spatial data, were applied for spatiotemporal evaluation and planning of vector control strategies. This approach has created new opportunities to enhance planning, management and monitoring capabilities related to health objectives by contributing to analysing the spatial relationships of factors and constraints in the implementation of public health programmes (Jaishankar and Jhonson, 2006). In this sense, GIS constitute a key procedure to evaluate activities and guide field campaigns efforts.

\section{Study area}

The work took place in General Taboada Department, Santiago del Estero Province, Argentina (28²7'32.64’'S, 6250'15.24”O). Data were analysed from four rural sites located less than $50 \mathrm{~km}$ from Añatuya City: El Desvío, Miel de Palo, La Salamanca and Pozo Herrera with a total population of 756 individuals divided between 209 households (Figure 1). Añatuya, the political, administrative and commercial centre of the region, has a population of 25.000 inhabiting approximately 6.500 dwellings (INDEC, 2010). The area is a transition between semi-arid and semi-humid Chaco forest, which presents extreme weather conditions such as summer temperatures reaching $50^{\circ} \mathrm{C}$. In winter, the average minimum temperature is $5^{\circ} \mathrm{C}$, while in summer the average maximum $34^{\circ} \mathrm{C}$.

The most common dwelling in the communities located in the study area is the rancho that has a complex peri-domestic backyard consisting of constructions by timber and bricks used as kitchen, animal pens (goats, chickens), an ideal habitat for the T. infestans vector. The houses in the area are arranged in a dispersed way and stand apart by distances ranging from hundreds of meters up to several $\mathrm{km}$.

\section{Data}

Working with the IChP in the rural zone of Añatuya, we focused on data related to house-level epidemiology, sociodemography and $T$. infestans infestation, including also other triatomine bugs. IChP had started in 2005 and the basic information of its reach is presented in Table 1 . We conducted 1,422 domiciliary visits during the time covering the consecutive cycles of intervention (C1 to C8) that ranged from May 2010 to June 2016. Site selections for the study were done in a way to obtain data homogeneity

Table 1. Overview of the integral Chagas programme.

\begin{tabular}{lcccc} 
Site & Number of houses & Number of inhabitants & Start date & Inhabitants* per house \\
El Desvío & 53 & 201 & Aug-05 & 3.8 \\
Miel de Palo & 81 & 314 & Jan-06 & 3.9 \\
\hline La Salamanca & 36 & 138 & Apr-07 & 3.8 \\
Pozo Herrera & 39 & 103 & Jun- 10 & 2.6 \\
\hline Total & 209 & 756 & - & - \\
\hline
\end{tabular}

*Average number. 
with respect to temporal coincidence.

Each house was georeferenced using Garmin Etrex or Legend Hcx GPS instruments and given an identification number, while the inhabitants received information and graphic material related to Chagas prevention. A house was considered positive for T. infestans if triatomine bugs (adults or nymphs) and/or their eggs were found during inspection. All findings were recorded and their specific locations (intra- and/or peri-domestic) were annotated and loaded onto a data management platform with a GIS designed and developed by the FMS information technology team. This platform included all households and the entomological results at each inspection, as well as control actions provided and socio-demographic information collected (number of inhabitants living in the home, ages of the inhabitants, schooling, level of education, social security, main economic activity of the family group, and characterization of housing construction and access to basic services).

The $T$. infestans infestation were defined and recorded throughout the study using the following entomological indices: i) intra-domestic infestation index (Int-Inf); ii) peri-domestic infestation index (Per-Inf); iii) presence of both intra- and peri-domestic infestation (Int-Per-Inf); and iv) presence of infestation either intra- and peri-domestic infestations (Tot-Inf), which were calculated by the formula $(i / t) * 100$, were $i$ is the infestation number (number of infested houses in the study area) and $t$ the total number of houses under investigation. A GIS was developed in order to collect all recorded intervention data.

Initial entomological evaluation was performed to estimate the $T$. infestans infestation baseline. In this initial visit, chemical control was subsequently performed in all houses (both at intra- and peridomestic structures) regardless of the outcome of the inspection according to the regulations of the National Ministry of Health (PNC, 2012). After the initial visit, a $S \& C$ system was established and each house from the five localities were inspected at least once every 12 months. The inspections were conducted by the hour/man method, in which two FMS-trained technicians inspected every house using an irritant spray ICONA-Espacial@ (Tetramethrin 0.2\%). The hour/man method consists in systematically, neatly and completely investigating the interior (intra-domicile) and the exterior (peridomicile) of a dwelling by a man for an hour to determine the presence of $T$. Infestans or other triatomines. If there are two people who evaluate the time it is reduced by half. The irritant is used as a tool to improve inspection in homes and in peridomiciliary structures.

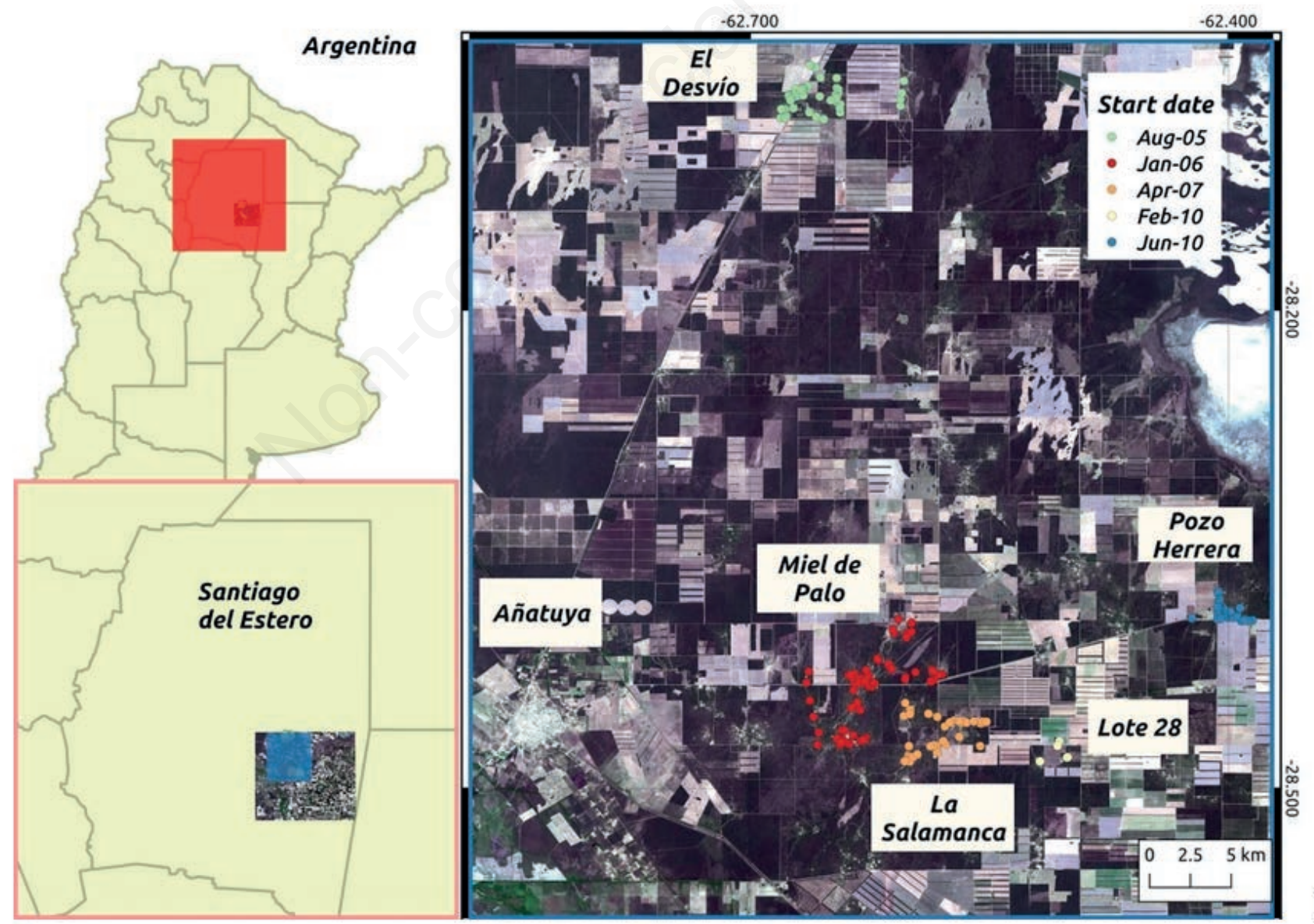

Figure 1. The study area. Copernicus Sentinel 2 data (2017) of the study area located less than $50 \mathrm{~km}$ from the Añatuya City, Santiago del Estero Province, Argentina (28 $\left.27^{\prime} 32.64^{\prime \prime} S, 62^{\circ} 50^{\prime} 15.24^{\prime \prime O}\right)$. 
It is applied to cracks and surfaces in order to cause triatomines to move. All intra-domestic environments and peri-domestic structures (fireplace, henhouse, deposits, kennels, animal pens, toilets or latrines, etc.) were inspected following National Ministry of Health recommendations (PNC, 2012).

After completion of a site inspection, the surveillance results were analysed and the intra- and peri-domestic areas chemically controlled by spraying of all houses with the following characteristics: i) presence of any stage of T. infestans; ii) houses found to be negative but located within a radius $<500 \mathrm{~m}$ from a positive house; and iii) other constructions with epidemiological, demographical or structural characteristics requiring control. Chemical control was performed using SIPERTRIN® (Chemotecnica, BetaCipermethrin 5\% (Asimethrin)), a liquid pyrethroid insecticide (50 $\mathrm{mg} / \mathrm{m}$ ) as defined by the National Ministry of Health (PNC, 2012).

To record the history of spraying and infestation used for S\&C spatial analysis of every house during each cycle of intervention (C1 to $\mathrm{C} 8$ ), we characterized each house by a binary variable, given below, indicating whether the house: i) was intra-domiciliary infested each time it was controlled (infestation_intra); ii) was peri-domiciliary infested each time it was controlled (infestation_peri); or iii) had been sprayed (spraying).

In addition, the following two variables were used to characterize the dwellings: i) distance from each house to the nearest house sprayed (dist_to_spr); and ii) distance from each house to the nearest house infested (dist_to_inf).

\section{Descriptive statistics}

Descriptive statistics analysis of the work corresponding to the different intervention cycles was carried out using $\mathrm{R}$ statistical software, based on the S\&C data. For the descriptive functions, only total infestations (presence of either intra- or peri-domestic infestation) were analysed.

Bi-dimensional histograms were implemented as a tool to describe the spatiotemporal patterns of both infestation and insecticide spraying where the spatial distance between house $n$ and house $m\left(\Delta X_{n, m}\right)$ is defined as (Eq. 1):

$\Delta X_{n, m}=X_{n}-X_{m}$ and $n, m \quad\left[1, \ldots, N_{h}\right]$

where $X$ represents distance and $N_{h}$ the total number of houses. Likewise, the temporal distance between the control time $i$ and the control time $j\left(\Delta T_{i j}\right)$ is defined as (Eq. 2):

$\Delta T_{i, j}=T_{i}-T_{j}$

where $i, j \quad[1, \ldots, L]$ and $L$ is the total number of controls.

For the infestation part of the study, let $I_{t d}$ and $I_{s d}$ be equal to arrays containing the temporal distances between all pairs of infested houses and the spatial distance between all pairs of infested houses, respectively. Then, the bi-dimensional histogram of infestation distances represents the two-dimensional density function of the two variables defined as (Eq. 3):

$\operatorname{Inf}_{2 d}=\operatorname{hist}_{2 d}\left(I_{t d} I_{s d}\right)$

Eq. 3

where $\operatorname{Inf}_{2 d}(i, j)$ is equal to the number of simultaneous occurrences of an element of $I_{t d}$ falling in the $i$ th bin, with the corresponding element of $I_{s d}$ falling in the $j$ th bin (i.e. representing the frequency of infested houses pairs separated by the temporal distance $i$ and the spatial distance $j$.

For the insecticide spraying part of the study, let $S_{t d}$ and $S_{s d}$ be arrays containing the following variables: temporal distance between all pairs of sprayed houses, $S_{t d}$, and spatial distance between all pairs of sprayed houses $S_{s d}$. Then the bi-dimensional histogram of spraying distances is defined as (Eq. 4):

$\operatorname{Spr}_{2 d}=h i s t_{2 d}\left(S_{t d} S_{s d}\right)$

Eq. 4

$\operatorname{Spr}_{2 d}(i, j)$ is equal to the number of simultaneous occurrences, or co-occurrence, of an element of $S_{t d}$ falling in the $i$ th bin, with the corresponding element of $S_{s d}$ belonging to the $j$ th bin (i.e. representing the frequency of sprayed houses pairs separated from among them for a temporal distance in the range of $i$ and a spatial distance in the range of $j$ ).

In order to exemplify this evaluation alternative, they were analysed two places: Miel de Palo and La Salamanca. Spatiotemporal pattern of infestation at two times: initial (the first 3 cycles of the programme) vs final (the last 3 programme cycles) it was compared, according to each spatial-temporal pattern of spraying.

\section{Infestation prediction by machine learning: use of neu- ral network}

The outcome variable peri-domestic infestation at intervention time 8 (infestation_peri) was modelled according to the neural network (NNET) model. The following S\&C spatial predictor variables: spraying, infestation_intra, infestation_peri, dist_to_spr, dist_to_inf of intervention times 1 to 7 were all examined according to the NNET model.

A NNet is an information-processing system that is made up of interconnected neurons, or interconnected information processing units in analogy with the human nervous system. The information processing units do not work in a linear manner. In fact, NNets draw the strength from parallel processing of information, which allows dealing with non-linearity. A NNet are useful with respect to the understanding of meaning and patterns generated by complex datasets. NNets are built by a massive number of simple processing units highly interconnected. They can be trained to provide universal function approximators. Nonlinear models are able to capture more complex functional relations among the data, at the expense of computational complexity and some burden on the user that has to fine tune more parameters than in linear models.

A NNet is a model characterized by an activation function, which is used by interconnected information processing units to transform input into output. The first layer of the NNet receives the raw input, processes it and passes the processed information to the hidden layers. The hidden layer passes the information to the last layer, which produces the output. The advantage of a neural network is that it is adaptive in nature. It learns from the information provided, i.e. trains itself from the data, which has a known outcome and optimizes its weights for a better prediction in situations with unknown outcome.

A perceptron, a single layer NNet, is the most basic form of a NNet. A perceptron receives multidimensional input and processes it using a weighted summation and an activation function. It is trained using a labelled data and learning algorithm that optimize the weights in the summation processor. A major limitation of the perceptron model is its inability to deal with non-linearity. A multilayered NNet overcomes this limitation and helps solve nonlinear problems. The input layer connects with a hidden layer, which in turn connects to the output layer. The connections are weighted and weights are optimized using a learning rule. 
Typically, machine learning regression includes three steps: i) architecture choice, e.g. the number of layers and neurons in an artificial neural network; ii) the training-validation step (where the coefficients are adjusted and the performance is evaluated); and then iii) the use of the model with new data. The scaling of data is essential because otherwise a variable may have large impact on the prediction variable only because of its scale. Using un-scaled may lead to meaningless results. The common techniques to scale data are: min-max normalization, Z-score normalization, median and median absolute deviation, and tan-h estimators. The min-max normalization transforms the data into a common range, thus removing the scaling effect from all the variables.

The implementation of the NNET model was carried out in the statistical software R. The following specifications of this implementation were:

- Standardization or scaling of the predictors in the [0-1] interval by leveraging the lapply function: scl<- function $(x)\{(x-$ $\min (x)) /(\max (x)-\min (x))\}$;

- Setting the argument linear.output to FALSE in order to tell the model that we want to apply the activation function (act.fct), since we are not doing a regression set task;

- Setting the activation function to logistic (which is the default option) in order to apply the logistic function;

- Application of the Iterated Racing for Automatic Algorithm Configuration (iRace) package: The configuration or selection of the optimal set of parameters (alpha, layers, neurons) in this kind of nonlinear models is a complex issue and could be handcrafted or obtained using semi-automatic tools. Following the procedure exposed in Scavuzzo et al., 2018, we used the iRace package (López-Ibáñez et al., 2016) for automatic parameter tuning. This tool is an iterative procedure capable of automatically finding the most appropriate parameter configurations given the input data instances of the optimization problem. It is implemented in $\mathrm{R}$ and is freely available at http://iridia.ulb.ac.be/irace/;

- Cross-validation (Cramer et al., 2017) to decrease the dependency of the evaluation results on a particular selection of training set and validation set pair. In all the cases, we generated the models with $80 \%$ of the dataset (training) and retained the remaining $20 \%$ as the independent set to corroborate the prediction capacity of the model (testing). We crossvalidated the model using 50 replicates of cross-validation with this $80 \% / 20 \%$ split, which is the most common proportions used (Cramer et al., 2017).

\section{Results}

The entomological indices collected at times covering the intervention cycles ranging from 2010 to 2016 are shown in Figure 2. Table 2 shows a summary of the infestation values for each site throughout the period analysed. The maximum values of total infestations (50), mean total infestation (20.82), infestation variability (15.3), and mean peridomestic infestation (16.58) were seen in La Salamanca. Pozo Herrera had the minimum mean of total infestation (13.07) and Miel de Palo the minimum variability of total infestation (standard deviation=4.87). Miel de Palo showed the maximum mean of intra-domestic infestation (4.94).

At the last control, all sites except El Desvío showed a reduction of infestations with respect to the initial value. At this village, and also at Pozo Herrera, intra-domestic infestation was low and did not seem to correspond to the dynamics of peri-domiciliary infestation. The site with the highest peri-domestic infestation in the initial period was Pozo Herrera (28.1), while the site with the highest intra-domestic infestation in this period was Miel de Palo (11.1). The only site with decreased peri-domestic infestation towards the end of the study period was Pozo Herrera, the rest of the sites increased peri-domestic infestation. In general, the sites did not modify intra-domestic infestation. About 33\% of infestations in each cycle were due to persistent infestation despite spraying (Figure 3). The rest of the infestations were possibly due to colonization (houses with new infestations) from the environment. In cases of houses that had no infestation in the previous time and were infested, previous, preventive spraying had been applied $14 \%$ of the time, so in these cases the insecticide would have no residual effect. The average distances of a house to an infested house and to a sprayed house, at the previous time, were lower when they were not infested $(1,000 \mathrm{~m})$ as compared to when they were infested $(1,500 \mathrm{~m})$. From the total number of times that an infested house was sprayed, $32 \%$ were not effective, since immediately after the intervention, those houses were re-infested. Of the total number of times that a non-infested dwelling was sprayed, $12 \%$ did not prevent colonization, confirming that in those cases there was no residual effect of the insecticide. After generating the variables described in the methodology section for each S\&C cycle, we proceeded to analyse the spatiotemporal patterns of these variables graphically using two tools: the scatter plot (Figure 4) and a twodimensional histogram (Figure 5). Figure 4 shows a scatter plot of the minimum distance to the infestation ( $\mathrm{x}$ axis) and the minimum distance to the spraying (y axis) in the time before a house was infested (left graph) with respect to the houses that they were not infested (right graph).

Table 2. Summary of the infestation values for each site throughout the period analysed.

\begin{tabular}{lccccc} 
Site & Max total & Mean total & SD total & Mean peri. & Mean intra. \\
El Desvío & 25.53 & 16.11 & 7.64 & 15.57 & 15.57 \\
Miel de Palo & 28.38 & 19.01 & 4.87 & 16.58 & 4.94 \\
\hline La Salamanca & 50 & 20.82 & 15.34 & 12.72 & 4.6 \\
Pozo Herrera & 28.13 & 13.07 & 10.28 & 0.71 \\
\hline
\end{tabular}

Max total, maximum of the total infestation indices; Mean total, mean of the total infestation indices; SD total, standard deviation (of the total infestation indices); Mean peri., mean of peridomestic infestation indices; Mean intra., mean of intra-domestic infestation indices. 
There were very few cases in which the distance to an infested house was less than the distance to a sprayed one (left of the diagonal). This is due to the spray strategy carried out by FMS. In those cases, the dwellings located at those distances of spraying and infestation at the time of the following intervention were mostly not infested.

The houses located on the diagonal in Figure 4A were the ones that were infested and being so at the time of previous intervention - they had the closest infested dwelling that was also sprayed. To the right of the diagonal were those infested, being that in the time of previous intervention they had the closest infestation at a distance greater than the closest spray. The percentages presented in Figure 3 are distributed in Figure 4A as follows: $33 \%$ of the infested dwellings are at point 0.0 of Figure $4 \mathrm{~A}$ (houses that were infested despite the fact that they were previously infested and sprayed),
$9 \%$ should be on the $\mathrm{x}$-axis (at a closer distance of spraying at the previous time equal to 0 ), $58 \%$ is distributed on the rest of the graph. Most houses are below the diagonal, that is, they have a closer spray prior to a shorter distance than the closest previous infestation. But they seem to be infested or not infested in an unstructured way, according to a random pattern.

Figure 5 shows the two-dimensional histograms of the minimum distance to the infestation ( $\mathrm{x}$ axis) and the minimum distance to the spraying (y axis) in the time before a house was infested (left graph) with respect to the houses that they were not infested (right graph). Counts indicates the number of dwellings that were infested (left graph) or not infested (right graph) with each distance characteristic (spraying and infestation) in the previous intervention time. The largest number of homes that were infested present in the previous time an infestation and sprayed at $500 \mathrm{~m}$. While those that
$\mathbf{A}$

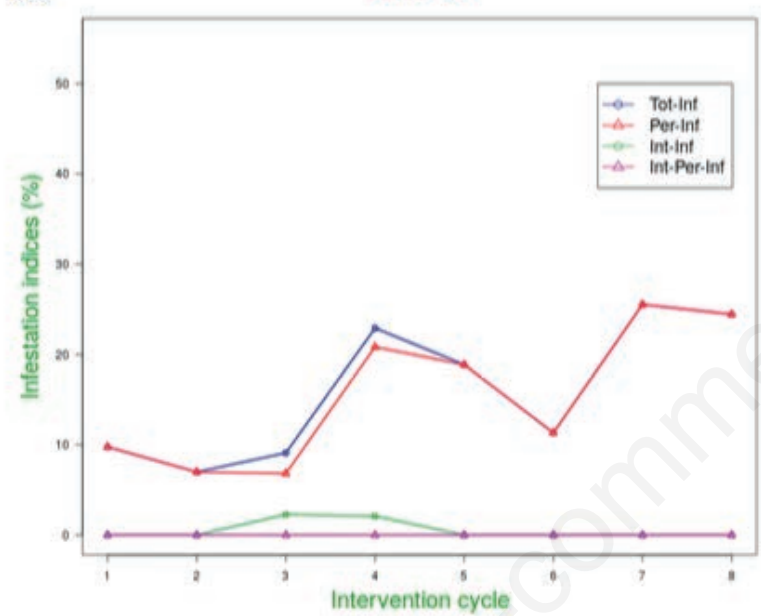

C

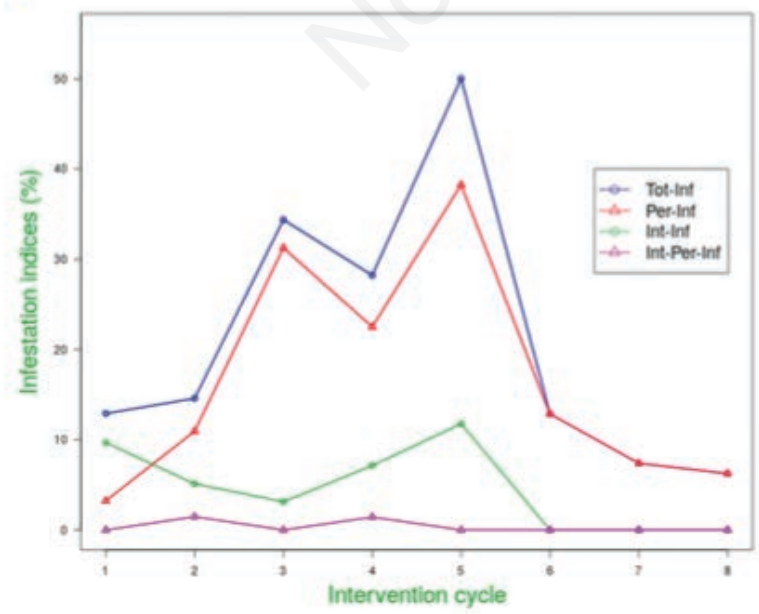

B

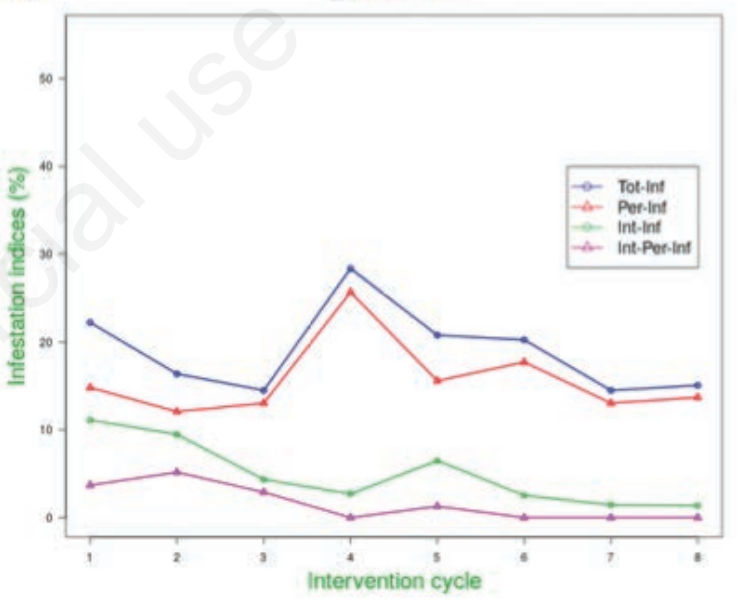

D

Pozo Herrera

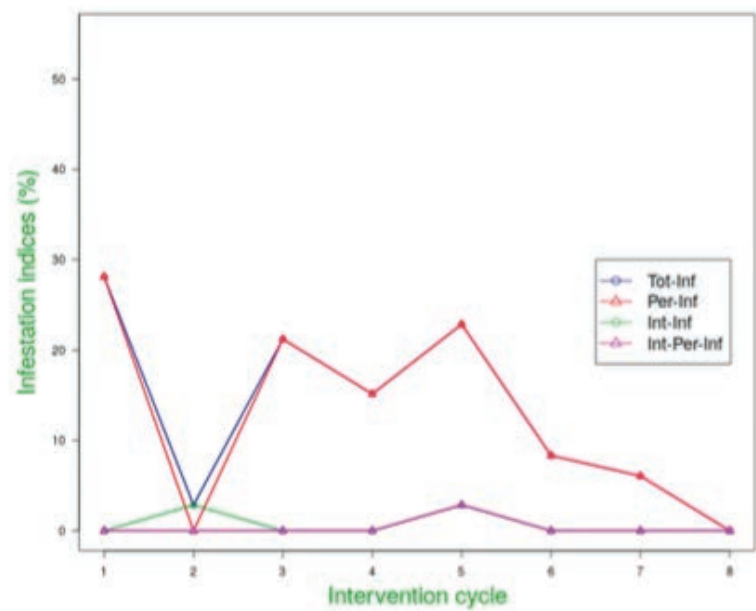

Figure 2. Entomological indices computed for each site over the study period. A) El Desvío; B) Miel de Palo; C) La Salamanca; D) Pozo Herrera. Tot-Inf, total infestation; Per-Inf, peri-domestic infestation; Int-Inf, intra-domestic infestation; Int-Per-Inf, both intra- and peri-domestic infestation. 
the largest number of homes that were not infested have homes infested and sprayed at a smaller distance (about $300 \mathrm{~m}$ ).

In Figure 6 the spatial-temporal pattern of intra- and peridomestic infestation is approached through bi-dimensional histograms. This represent the frequency of infested houses pairs separated from among them for each temporal and spatial distance. Figure 6 therefore presents $2 D$-histograms showing the spatialtemporal pattern of infestation (initial $v s$ final) and spraying strategies of two places: Miel de Palo and La Salamanca.

In Miel de Palo, the initial pattern of infestation (with a peak of infested houses separated at a spatial distance of 4,000 m and timewise by 9 months) seemed to be conserved at the final time but decreasing the number of cases of infestation by $30 \%$. At the end at the La Salamanca site, the peak number of cases located in the same intervention time was eliminated and the peak of cases separated by 9 months of temporal distance was conserved, but the same happened to be around 1,000 $\mathrm{m}$ further away. The analysis of the spraying patterns in both places led us to conclude that the spray pattern in La Salamanca had a spraying peak at lower spatial distances than the peak of initial infestations. By contrast, in Miel de Palo, the spraying peak occurred at spatial distances greater than its corresponding spatial distance of infestation at the initial time. Apparently in La Salamanca, the spray pattern would have an effect of decreasing infestation at close spatial distances more marked than in Miel de Palo.

Regarding the results of the computed NNET model, the implementation of the iRace tool resulted in the best configuration of neurons and layers for our model of 10 neurons in its 2 hidden layers.

The weights of NNET model were calculated using the backpropagation algorithm. Cross-validation of our implemented model resulted in a mean accuracy of 0.77 . In Figure 7 the predicted peridomestic infestation at C9 based on the fitted NNet model is shown with respect to $\mathrm{S} \& \mathrm{C}$ spatio temporal variables of intervention times $\mathrm{C} 2-\mathrm{C} 8$.

\section{Discussion}

Control strategies based on spraying with insecticides have made very important contributions in controlling Chagas in Latin
America (Dias et al., 2008). However, it is important to know where and when to spray. Re-infestation is sensitive to environmental variables, but in this study we focused on exploring its relationship with variables linked to control activities, such as spatial and temporal distances to the closest sprayed house. This paper describes the result of work within an area endemic for Chagas with a known case history. It involves interventions by control
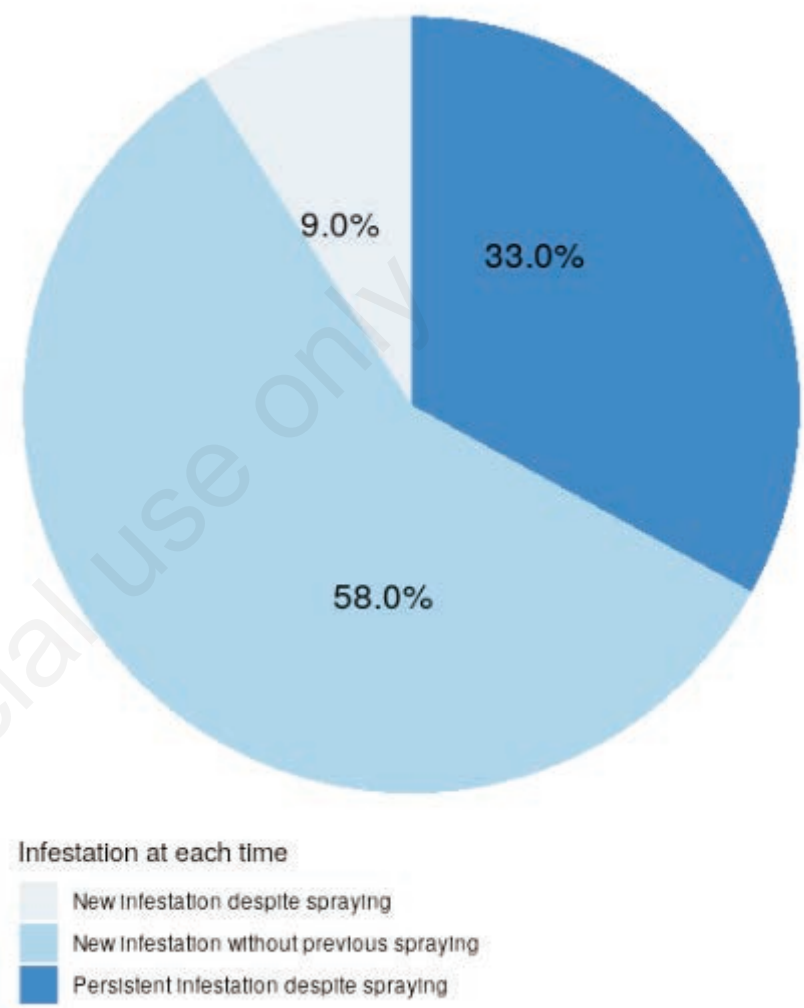

Figure 3. The infestation situation over the whole time of the study analysed by descriptive statistics at each time of intervention.
A

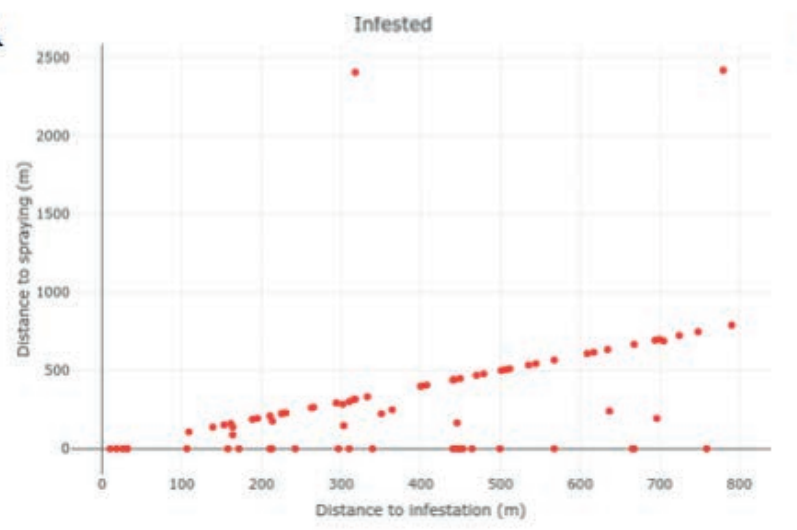

B

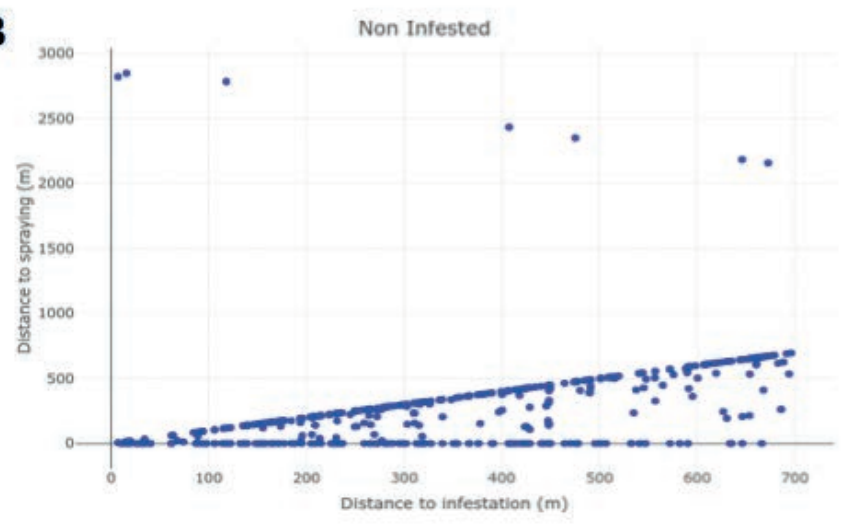

Figure 4. Scatter plot of minimum distance to infestation and spraying. A) infested houses; B) non-infested houses. 
agents who, after detecting the presence of $T$. infestans, proceeded to spraying with deltamethrin according to WHO recommendations. Emphasis was placed on the possibility of extracting as much information as possible about this intervention through spatiotemporal analysis based on geospatial tools and the work constitutes an attempt to explain the effect of the S\&C interventions, sus- tained by the control programme over six years

We demonstrate a useful and practical method for systematic insecticide spraying based on documented previous infestation and spraying. In terms of overall evaluation of the analysed intervention period, most sites presented less infestations, in fact only minor ones, at the latest control included in the study compared to the initial one.
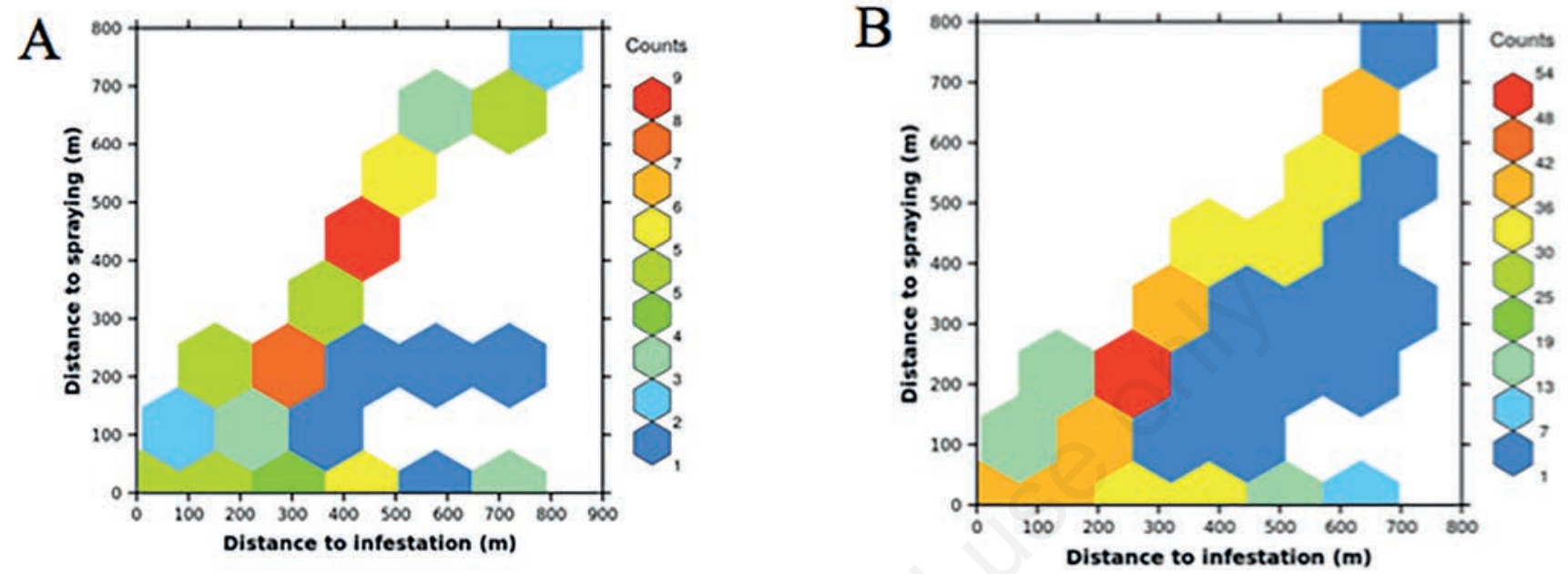

Figure 5. Two-dimensional histograms of minimum distance to infestation and spraying. Counts indicates the number of dwellings that were infested (A, left graph) or not infested (B, right graph) with each distance feature (spraying and infestation) at the previous intervention time.

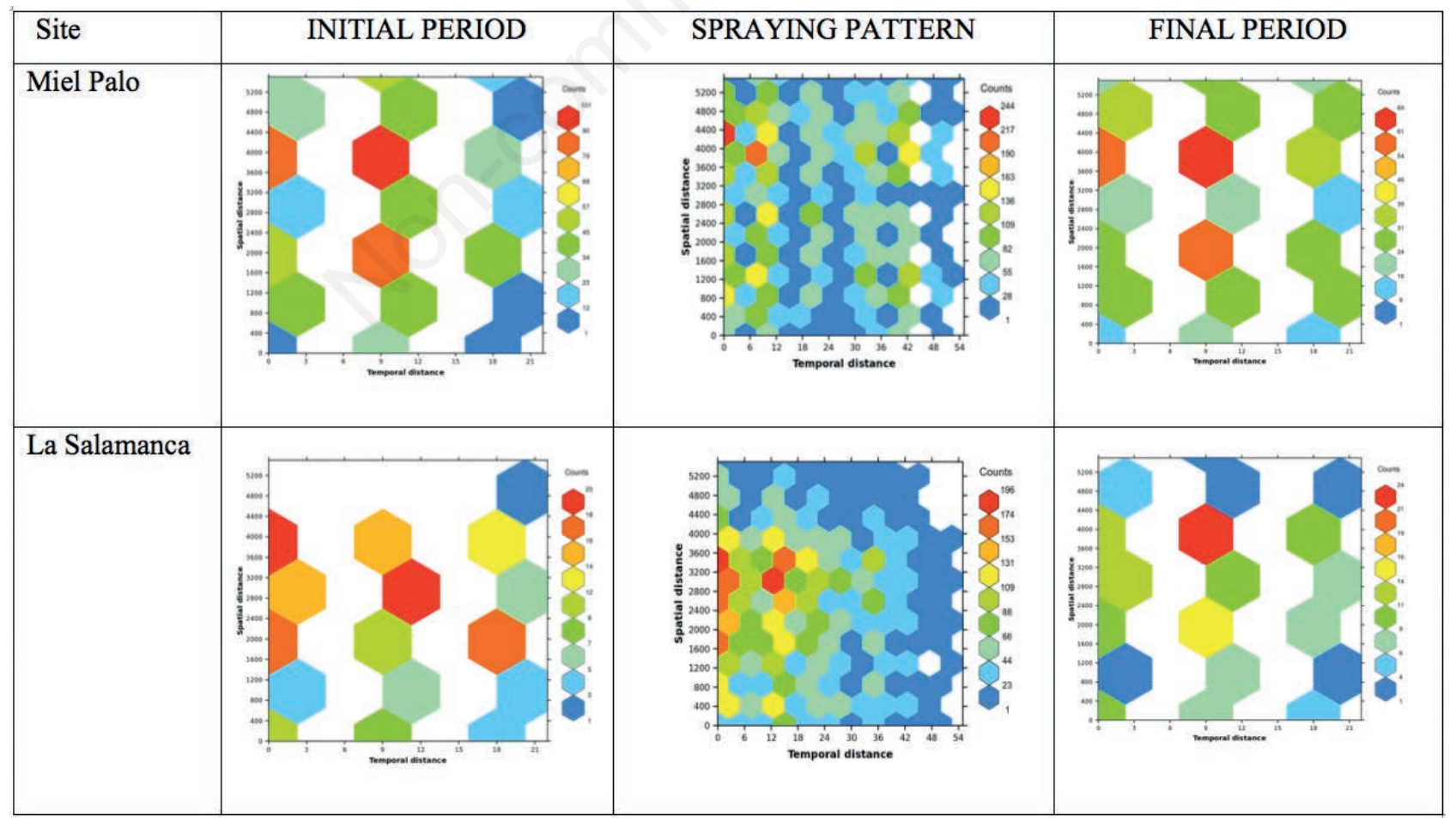

Figure 6. Bi-dimensional histograms showing the spatial-temporal pattern of infestation (initial $v s$ final period) and spraying at two places: Miel de Palo and La Salamanca. Spatial distance expressed in meters and temporal expressed in months. 
This was mainly due to a decrease in intra-domestic infestations. However, since there was no experimental design in the sense allowing comparison of places with vigilance to others without control. Instead, we tried to capture the information contained in control variations estimated through spatial indices related to spraying and infestation already implemented.

In spite of the overall results, we as much as about $33 \%$ of persistent infestations despite spraying, possibly due to re-colonization of $T$. infestans from the environment. The fact that preventive spraying had no residual effect in so many cases as also observed by multiple studies (Cecere et al., 2003; Spaugnolo et al., 2011). The widespread demonstration of triatomines survival despite insecticide spraying is a worry, even though there has been a $70 \%$ decrease in new infections in the last decade (Moncayo, 2003). Indeed, as confirmed by our work, vector control without reservoir control measures is not sufficient to reduce triatomine persistence. Comprehensive programmes involving house improvements, active participation and multi-sectoral coordination is required (De Urioste-Stone et al., 2015). Elimination of Chagas might also necessitate more efficient drugs and the development of an effective vaccine as called for by Moncayo, 2003.

By the realization of improved rural dwellings with enhanced sanitary quality based on adequate technology and locally available material, the FMS programme have contributed to villagers' well-being and social progress in Añatuya. However, prevention of Chagas also requires intersectoral and multi-disease approaches. Therefore, integration of S\&C activities into the local primary health care system at the community level would be useful. Thus, schools and community networks can become key strategies to reach Chagas disease elimination. As shown by our results, the FMS complementary programme has already had a big impact in this context.

The largest number of homes that were not infested at each intervention cycle had homes infested and sprayed at a smaller distance $(150-300 \mathrm{~m})$ in the previous cycle, compared to houses that were infested. This could indicate that in order to be free from infestation, a spraying at least $300 \mathrm{~m}$ away from each infested house would have to be guaranteed. In the same way, in terms of qualitative evaluation of spray strategies using spatiotemporal graphic tools, it is possible to hypothesize that a spray pattern that peaks at lower spatial distances than the peak of infestations, would have an effect of more marked decreasing infestation at closer spatial distances.

The application of GIS for spatial analysis has significant importance for vector control programmes, and new analytics approaches can be useful in order to improve control efficiency. Chu et al. (2013) have contributed to the evaluation and planning of control strategies using GIS. However, reports evaluating control programmes that consider the spatiotemporal history of infestation and spraying of a site are generally lacking in the literature. Although the evaluation proposed in this work is qualitative, it could be the first step towards a quantitative validation using the spatiotemporal structure of spraying to investigate the distribution of infestation.

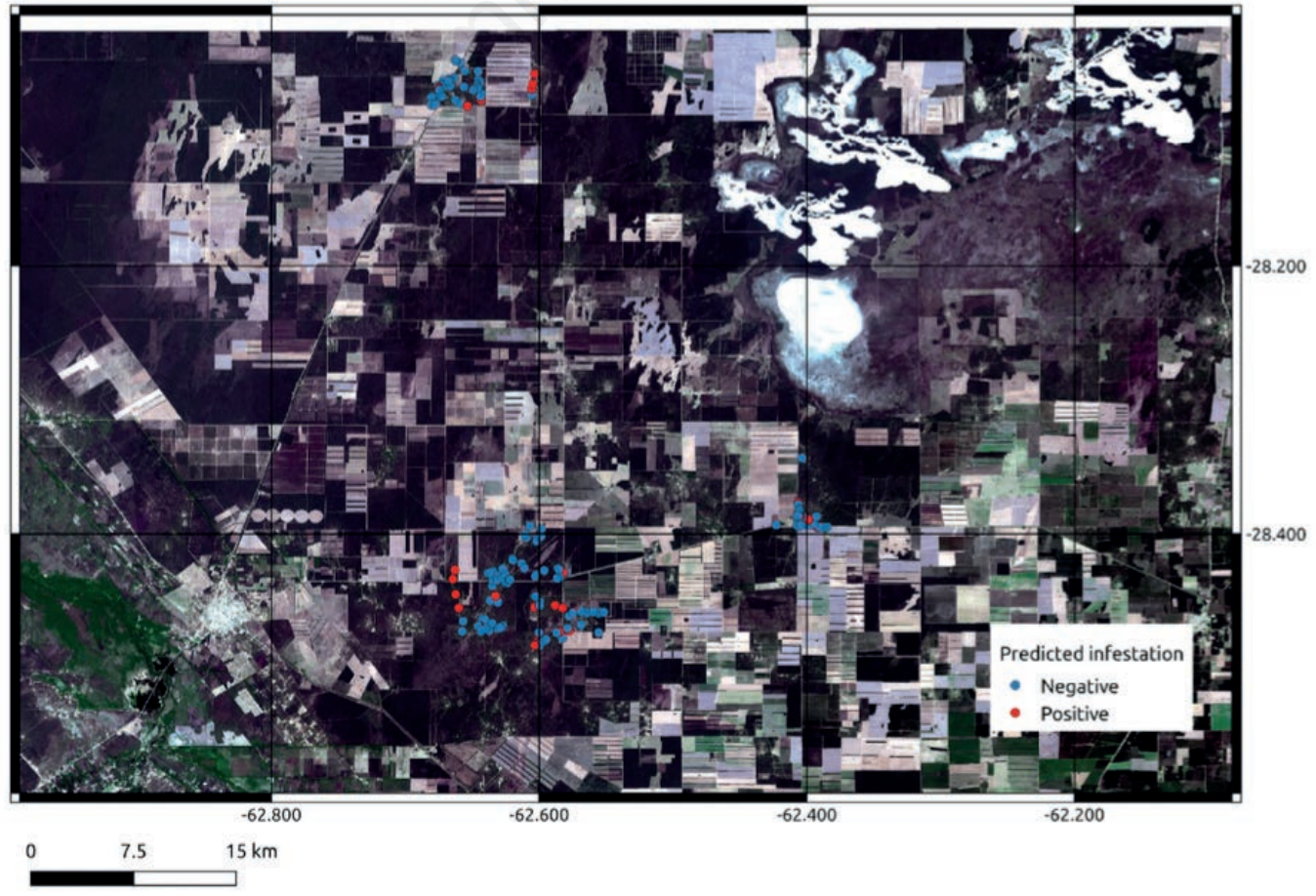

Figure 7. Predicted peri-domestic infestation at C9 based on the fitted neural network model and the surveillance and control spatiotemporal variables at intervention times $\mathrm{C} 2-\mathrm{C} 8$. 


\section{Conclusions}

This work demonstrates that tools can be developed and introduced to extract information from existing data. It presents new ways of visualizing spatiotemporal information achieved not only by qualitative evaluation of traditional, entomological indices or the distribution of infested houses, but through investigating the spatiotemporal pattern of infestations and spraying. This is important as it becomes possible to evaluate and plan spray strategies based on predictions of infestation from novel, non-linear models using the spatiotemporal indices implemented.

\section{References}

Abad-Franch F, Diotaiuti L, Gurgel-Goncalves R, Gurtler RE, 2013. Certifying the interruption of Chagas disease transmission by native vectors: cui bono? Mem Inst Oswaldo Cruz 108:251-54.

Abril M, Coto H, Weinberg D, Coppede ME, Cejas RG, 2009. Mejoramiento de viviendas con participación comunitaria para la prevención y el control de la enfermedad de Chagas en comunidades rurales del sureste de Santiago del Estero, República Argentina. Emf Emerg 11:28-33.

Cecere MC, Canale DM, Gurtler RE, 2003. Effects of refuge availability on the population dynamics of Triatoma infestans in central Argentina. J Appl Ecol 40:742-51.

Chu HJ, Chan TC, Jao FJ, 2013. GIS-aided planning of insecticide spraying to control dengue transmission. Int J Health Geogr $12: 42$.

Cramer S, Kampouridis M, Freitas AA, Alexandridis AK, 2017. An extensive evaluation of seven machine learning methods for rainfall prediction in weather derivatives. Expert Syst Appl 85:169-81.

De Urioste-Stone SM, Pennington PM, Pellecer E, Aguilar TM, Samayoa G, Perdomo HD, Enríquez H, Juárez JG, 2015. Development of a community-based intervention for the control of Chagas disease based on peridomestic animal management: an eco-bio-social perspective. Trans R Soc Trop Med Hyg 109:159-67.

Dias JC, Prata A, Correia D, 2008. Problems and perspectives for Chagas disease control: in search of a realistic analysis. Rev Soc Bras Med Trop 41:193-6.

Dias JCP, 2002. O controle da doença de Chagas no Brasil. In: Silveira AC, ed. El Control de la Enfermedad de Chagas en los Países del Cono Sur de América. Historia de una Iniciativa Internacional, 1991/2001. Faculty of Medicine, Triângulo Mineiro, Uberaba, Brazil, pp 145-250.

Dias JCP, Schofield CJ, 1999. The evolution of Chagas disease (American Trypanosomiasis) control after 90 years since Carlos Chagas discovery. Mem Inst Oswaldo Cruz 94:103-21.

Gorla DE, Vargas Ortiz R, Catalá SS, 2015. Control of rural house infestation by Triatoma infestans in the Bolivian Chaco using a microencapsulated insecticide formulation. Parasite Vector $8: 255$.

Gürtler RE, Canale DM, Spillmann C, Stariolo R, Salomón OD, Blanco S, Segura EL, 2004. Effectiveness of residual spaying of peridomestic ecotopes with deltamethrin and permethrin on
Triatoma infestans in rural western Argentina: a district wide randomized trial. Bull WHO 82:196-205.

INDEC, 2010. Censo nacional de población, hogares y viviendas 2001 y 2010. National Institute of Statistics and Census of the Republic of Argentina, Buenos Aires, Argentina.

Jaishankar R, Jhonson CP, 2006. Geomatics and public health. Indian J Public Health 50:24-7.

López-Ibáñez M, Dubois-Lacoste J, Pérez Cáceres L, Birattari M, Stützle T, 2016. The irace package: iterated racing for automatic algorithm configuration. Operat Res Perspect 3:43-58.

Moncayo A, 2003. Chagas disease: current epidemiological trends after the interruption of vectorial and transfusional transmission in the Southern Cone countries. Mem Inst Oswaldo Cruz 98:577-91.

Moreno ML, Hoyos L, Cabido M, Catalá SS, Gorla DE, 2012. Exploring the association between Trypanosoma cruzi infection in rural communities and environmental changes in the southern Gran Chaco. Mem Inst Oswaldo Cruz 107:231-7.

PNC, 2012. Programa nacional de Chagas, Ministerio de Salud de la Nación. Guía para el control vectorial de la enfermedad de Chagas. National Ministry of Health, Argentina.

Remme JHF, Feenstra P, Lever PR, Medici AC, Morel CM, Noma M, Ramaiak K, Richards F, Sketeli A, Schmunis G, Van Brakel WH, Vassall A, 2006. Tropical diseases targeted for elimination: Chagas disease, lymphatic filariasis, onchocerciasis and leprosy. In: Jamison DT, Breman JG, Measham AR, eds. Disease control priorities in developing countries. 2nd ed. World Bank, Washington, DC/Oxford University Press, New York, pp 433-449.

Scavuzzo JM, Trucco F, Espinosa M, Tauro CB, Abril M, Scavuzzo CM, Frery AC, 2018. Modeling dengue vector population using remotely sensed data and machine learning. Acta Trop 185:167-75.

Schofield CJ, Jannin J, Salvatella R, 2006. The future of Chagas disease control. Trends Parasitol 22:583-8.

Silveira A, Vinhaes M, 1999. Elimination of vector-borne transmission of Chagas disease. Mem Inst Oswaldo Cruz 94:40511.

Spagnuolo AM, Shillor M, Stryker GA, 2011. A model for Chagas disease with controlled spraying. J Biol Dynam 5:299-317.

Waleckx E, Gourbière S, Dumonteil E, 2015. Intrusive triatomines and the challenge of adapting vector control practices. Mem Inst Oswaldo Cruz 110:324-38.

Weinberg D, Porcasi X, Lanfri S, Abril M, Scavuzzo CM, 2018. Spatial analysis of triatomine infestation indices and their association to the actions of a Chagas disease program and environmental variables during a 5-year intervention period. Acta Trop 188:41-9.

WHO, 2003. The World Health Report 2003, annex 2. Deaths by cause, sex and mortality stratum in WHO regions, estimates for 2002. World Health Organization, Geneva, Switzerland. Available from http://www.who.int/whr/2003/en/Annex2en.pdf Accessed: November 21, 2005.

WHO, 2016. Chagas disease (American trypanosomiasis). Fact sheets. World Health Organization, Geneva, Switzerland.

WHO, 2019. Neglected tropical diseases. World Health Organization, Geneva, Switzerland. Available from: www.who.int/neglected_diseases/diseases/en/ 